\title{
Solitary Peutz-Jeghers type hamartomatous polyps in the duodenum are not always associated with a low risk of cancer: two case reports
}

Yusuke Sekino ${ }^{1}$, Masahiko Inamori ${ }^{*}$, Mitsuru Hirai ${ }^{2}$, Kaori Suzuki ${ }^{1}$, Kaoru Tsuzawa $^{2}$, Keiko Akimoto ${ }^{2}$, Ayako Takahata², Nobutaka Fujisawa², Kumiko Saito², Akisa Tsunemi ${ }^{3}$, Michio Tanaka ${ }^{3}$, Hiroshi lida', Yasunari Sakamoto', Hirokazu Takahashi', Tomoko Koide', Chikako Tokoro', Yasunobu Abe', Atsushi Nakajima', Shin Maeda ${ }^{1}$ and Shigeru Koyama ${ }^{2}$

\begin{abstract}
Introduction: A hamartomatous polyp without associated mucocutaneous pigmentation or a family history of Peutz-Jeghers Syndrome is diagnosed as a solitary Peutz-Jeghers type hamartomatous polyp. As compared with Peutz-Jeghers Syndrome, Peutz-Jeghers type hamartomatous polyps are diagnosed with a lower risk of cancer and are regarded as a different disorder.

Case presentation: In case one, we describe an 84-year-old Japanese man with a $14 \mathrm{~mm}$ duodenal polyp. Endoscopic mucosal resection was performed and histological examination showed findings suggestive of a hamartomatous polyp with a focus of well-differentiated adenocarcinoma. In case two, we describe a 76-year-old Japanese man who had been treated for prostate, rectal and lung cancer. Upper gastrointestinal endoscopy revealed a duodenal polyp measuring $15 \mathrm{~mm}$ in diameter. Endoscopic mucosal resection was performed, and histological examination showed findings suggestive of a hamartomatous polyp. Liver and thyroid cancers were found after the endoscopic treatment.

Conclusion: Although duodenal solitary hamartomatous polyps are associated with a lower risk of cancer, four patients, including our cases, have been diagnosed with cancerous polyps. Patients with duodenal solitary hamartomatous polyps should be treated by endoscopic or surgical resection and need whole-body screening.
\end{abstract}

\section{Introduction}

Peutz-Jeghers Syndrome (PJS) is a rare autosomal dominant syndrome which is characterized by gastrointestinal hamartomatous polyps and mucocutaneous pigmentation [1,2], first described by Peutz in 1921 [3].

A hamartomatous polyp without associated mucocutaneous pigmentation or a family history of PJS is diagnosed as a solitary Peutz-Jeghers type hamartomatous polyp [4]. As compared with PJS, Peutz-Jeghers type hamartomatous polyps are diagnosed with a lower risk

\footnotetext{
* Correspondence: inamorim@med.yokohama-cu.ac.jp 'Gastroenterology Division, Yokohama City University School of Medicine, 39 Fukuura Kanazawa-ku, Yokohama, 236-0004, Japan Full list of author information is available at the end of the article
}

of cancer [5] and have been regarded as a different disorder from PJS.

We report two cases with a solitary Peutz-Jeghers type hamartomatous polyp treated by endoscopic mucosal resection.

\section{Case Presentations}

Case 1 is an 84-year-old Japanese man with previous medical history of hypertension, chronic hepatitis C infection, idiopathic thrombocytopenic purpura and colon polyps (tubular adenoma and tubulovillous adenoma). He had no mucocutaneous pigmentation or family history of PJS. An upper gastrointestinal endoscopy revealed a lobular polyp measuring $14 \mathrm{~mm}$ in

\section{() Biomed Central}




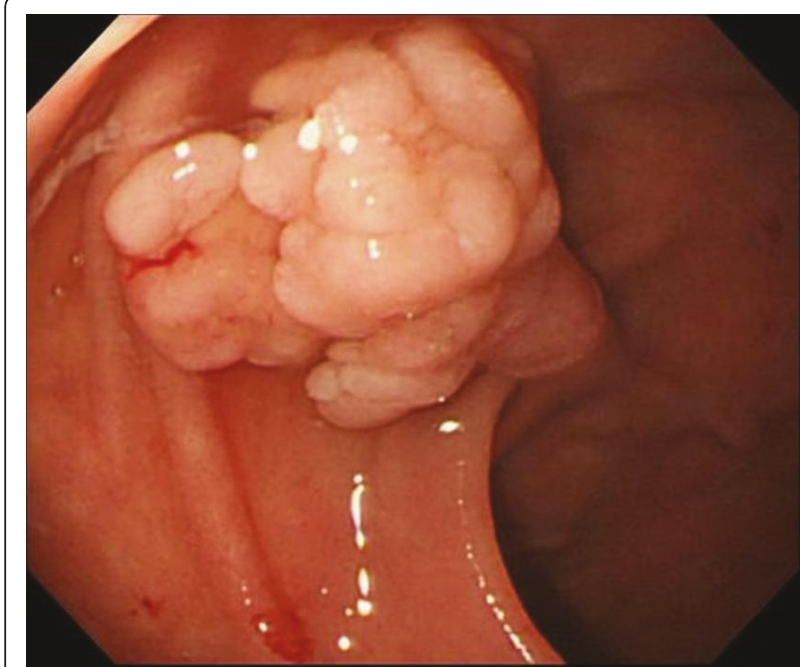

Figure 1 A lobular duodenal polyp measuring $14 \mathrm{~mm}$ in diameter was detected in the superior duodenal angle.

diameter, in the superior duodenal angle (Figure 1). Endoscopic mucosal resection was performed without complication and histological examination showed findings suggestive of a hamartomatous polyp-branching bundles of smooth muscle fibers covered by hyperplastic duodenal mucosa-with a focus of well-differentiated adenocarcinoma (Figure 2). A colonoscopy and smallintestinal follow-through showed no other polyps.

Case 2 is a 76-year-old Japanese man who had been treated for prostate, rectal and lung cancer, with no mucocutaneous pigmentation or family history of PJS. An upper gastrointestinal endoscopy revealed a

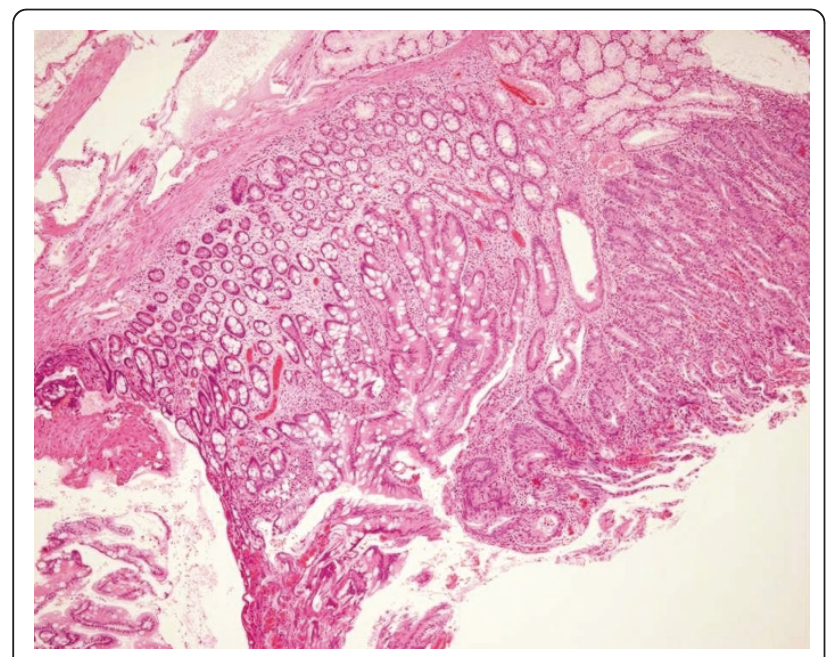

Figure 2 Histological examination showed findings suggestive of a hamartoma: branching bundles of smooth muscle fibers covered by hyperplastic duodenal mucosa, with a focus of well-differentiated adenocarcinoma. Hematoxylin and eosin staining (objective $10 \times$ ). duodenal polyp measuring $15 \mathrm{~mm}$ in diameter in the second part of his duodenum (Figure 3). Endoscopic mucosal resection was performed, and histological examination showed findings suggestive of a hamartomatous polyp (Figure 4). A colonoscopy and smallintestinal follow-through showed no other polyps. After the endoscopic treatment, concomitant liver and thyroid cancers were found.

\section{Discussion}

As compared with PJS, Peutz-Jeghers type hamartomatous polyps are diagnosed at a more advanced age, in the absence of mutation of the STK11/LKB-1 gene, and without familial history and mucocutaneous pigmentation [5].

Previous reports showed that polyps due to PJS had 3$6 \%$ of neoplastic change, such as adenomas or carcinomas [4-18]. A search of case reports on the MEDLINE database up to July 2010, using the terms "hamartomatous polyp" and "duodenum", and of reference lists of published articles (including our cases), showed 27 patients with a solitary Peutz-Jeghers type hamartomatous polyp in the duodenum (Table 1). Although solitary Peutz-Jeghers type hamartomatous polyps have been considered to show a lower potential for malignant transformation as compared to PJS, three cases (including ours) of solitary Peutz-Jeghers type hamartomatous polyps with malignant components have been reported since 2008, and the total malignant transformation rate of solitary Peutz-Jehgher type hamartomatous polyps was four out of $27(14.8 \%)$. There were no significant tendencies of malignant transformation within the age

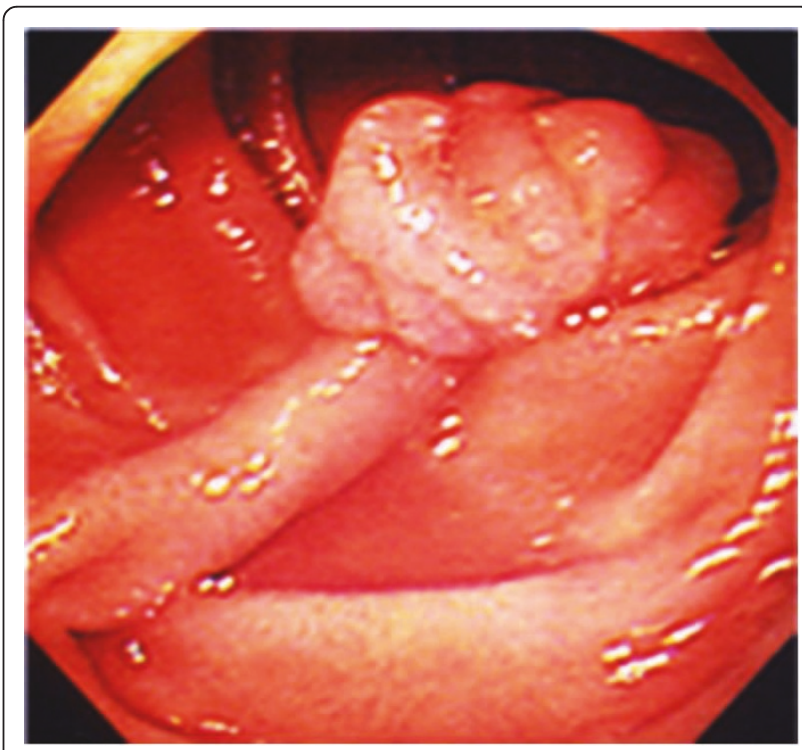

Figure 3 Pedunculated duodenal polyp measuring $15 \mathrm{~mm}$ in diameter in the second part of the duodenum. 


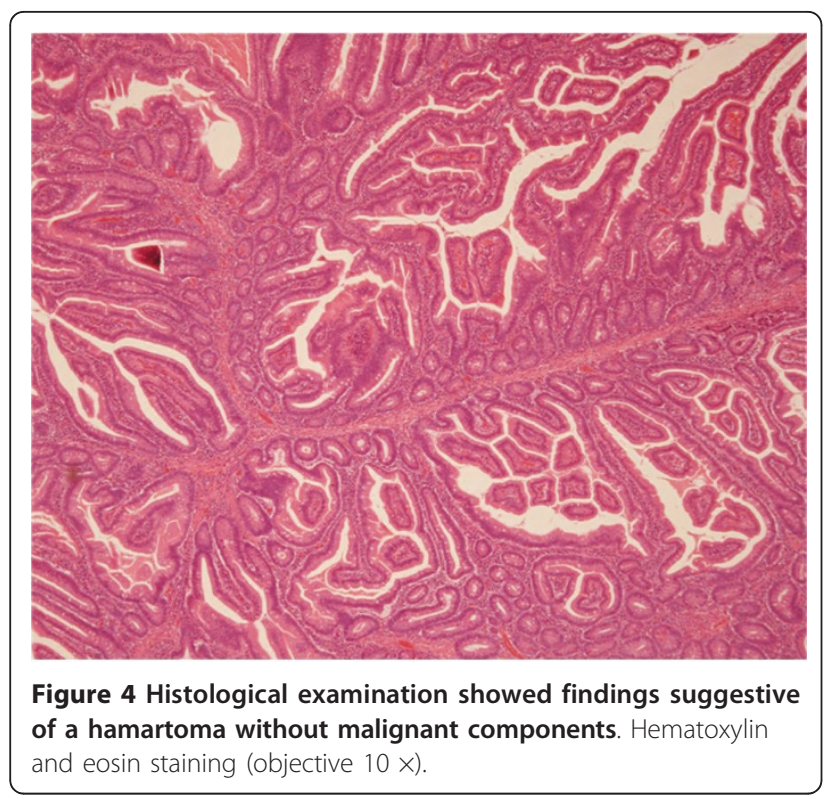

or sex of the patient, or the location, size or endoscopic appearances of the polyp.

The most serious problem in PJS is an increased risk of cancer in the gastrointestinal tract. The occurrence of cancer in the gastrointestinal tract has been reported in $20-25 \%$ of patients with PJS, and a risk of cancer in other organs has been also reported, including the ovary, breast, bladder, pancreas and thyroid [2,19-22].

To the best of our knowledge, there have been no previous reports of patients with solitary Peutz-Jeghers type hamartomatous polyps presenting with malignancy in other organs. This is one of the reasons that solitary Peutz-Jeghers type hamartomatous polyps have been considered as a separate clinical entity from PJS. However, Case 2 in our report had duplicated malignancy in six organs. An overlap between solitary Peutz-Jeghers type hamartomatous polyps and PJS may need to be reexamined.

Our two cases were diagnosed in patients with an advanced age similar to previous reports, but they differ in the malignant alteration of a hamartomatous polyp and concomitant other cancers. Patients with duodenal Peutz-Jeghers type hamartomatous polyps should undergo colonoscopy and whole-body screening; duodenal solitary Peutz-Jeghers type hamartomatous polyps should preferably be treated by endoscopic or surgical resection.

\section{Conclusions}

We report two cases of duodenal solitary Peutz-Jeghers type hamartomatous polyp. Case 1 was a hamartomatous polyp with a focus of well-differentiated adenocarcinoma, and Case 2 was a hamartomatous polyp with

Table 1 Twenty-seven cases of solitary duodenal Peutz-Jeghers type hamartomatous polyps.

\begin{tabular}{|c|c|c|c|c|c|c|c|c|c|}
\hline Author & Year & Number of patients & Age & Sex & Location & Surface & Size $(\mathrm{mm})$ & Treatment & Malignant transformation \\
\hline Gannon [6] & 1962 & 6 & NS & NS & NS & Smooth & NS & NS & No \\
\hline Shiegel [7] & 1978 & 1 & 75 & NS & 2nd & Smooth & NS & surgery & No \\
\hline Ushijima [8] & 1986 & 1 & 46 & M & 2nd & Lobulated & $20 \times 20 \times 15$ & endoscopy & No \\
\hline Bott [9] & 1986 & 1 & 23 & M & 4th & NS & $50 \times 40$ & surgery & No \\
\hline Naitoh [10] & 1988 & 1 & 56 & $\mathrm{~F}$ & $3 r d$ & Smooth & $30 \times 15$ & endoscopy & No \\
\hline Rossetti [11] & 1989 & 1 & 22 & $\mathrm{~F}$ & 2nd & Smooth & 50 & endoscopy & No \\
\hline \multirow[t]{2}{*}{ Tanaka [12] } & 1990 & 2 & 41 & M & $3 r d$ & Lobulated & $25 \times 18$ & endoscopy & No \\
\hline & & & 82 & $\mathrm{~F}$ & 2nd & Lobulated & $25 \times 20$ & endoscopy & No \\
\hline Nebri [4] & 1993 & 1 & 63 & $\mathrm{~F}$ & $1 s t$ & NS & $50 \times 35$ & surgery & No \\
\hline Ichiyoshi [13] & 1996 & 1 & 84 & $F$ & 2nd & Lobulated & $25 \times 20$ & endoscopy & Yes \\
\hline \multirow[t]{2}{*}{ Oncel [14] } & 2003 & 2 & 68 & $\mathrm{~F}$ & $3 r d$ & NS & 15 & endoscopy & No \\
\hline & & & 53 & M & $2 n d$ & Multiple polyps & 5 & endoscopy & No \\
\hline Kitaoka [5] & 2004 & 1 & 22 & $\mathrm{~F}$ & $1 s t$ & Lobulated & $35 \times 30 \times 30$ & endoscopy & No \\
\hline \multirow[t]{2}{*}{ Itaba [15] } & 2006 & 2 & 87 & $\mathrm{~F}$ & 2nd & NS & 17 & endoscopy & No \\
\hline & & & 56 & M & $1 \mathrm{st}$ & Lobulated & 12 & endoscopy & No \\
\hline \multirow[t]{3}{*}{ Suzuki [16] } & 2008 & 3 & 59 & $\mathrm{~F}$ & $2 n d$ & Lobulated & $15 \times 15$ & surgery & No \\
\hline & & & 68 & $\mathrm{~F}$ & 2nd & Lobulated & $10 \times 8$ & endoscopy & Yes \\
\hline & & & 60 & $\mathrm{~F}$ & $1 \mathrm{st}$ & Lobulated & $10 \times 10$ & endoscopy & No \\
\hline Jamaludin [17] & 2009 & 1 & 46 & M & $1 s t$ & Lobulated & $70 \times 40$ & surgery & Yes \\
\hline Kantarcioglu [18] & 2009 & 1 & 28 & $M$ & $2 n d$ & Lobulated & $25 \times 15$ & endoscopy & No \\
\hline \multirow[t]{2}{*}{ Sekino: our report } & 2010 & 2 & 84 & $M$ & $2 \mathrm{nd}$ & Lobulated & $16 \times 13$ & endoscopy & Yes \\
\hline & & & 76 & $M$ & $2 n d$ & Lobulated & 15 & endoscopy & No \\
\hline
\end{tabular}


five cancers in other organs. We advise that patients with duodenal solitary Peutz-Jeghers type hamartomatous polyps should preferably be treated with endscopic or surgical resection and whole-body screening.

\section{Consent}

Written informed consent was obtained from both patients for publication of these case reports and any accompanying images. Copies of the written consent are available for review by the Editor-in-Chief of this journal.

\section{Abbreviations}

PJS: Peutz-Jeghers Syndrome.

\section{Acknowledgements}

No funding was required for this study.

\section{Author details}

'Gastroenterology Division, Yokohama City University School of Medicine, 39 Fukuura Kanazawa-ku, Yokohama, 236-0004, Japan. ${ }^{2}$ Department of Gastroenterology, Tokyo Metropolitan Hiroo Hospital, 2-34-10 Ebisu, Shibuyaku, Tokyo, 150-0013, Japan. ${ }^{3}$ Department of Pathology, Tokyo Metropolitan Hiroo Hospital, 2-34-10 Ebisu, Shibuya-ku, Tokyo, 150-0013, Japan.

\section{Authors' contributions}

YS, MI, MH, KS, KT and KA analyzed and interpreted the patient data. AT, NF, KS, AT, MT and HI analyzed endoscopic data. YS, HT, TK, CT, YA, AN and SM performed the histological examination of the organs. YS, MI and SK were major contributors in writing the manuscript. All authors read and approved the final manuscript.

\section{Competing interests}

The authors declare that they have no competing interests.

Received: 11 November 2010 Accepted: 27 June 2011

Published: 27 June 2011

\section{References}

1. Jeghers $\mathrm{H}$, Mckusick VA, Katz KH: Generalized intestinal polyposis and melanin spots of the oral mucosa, lips and digits; a syndrome of diagnostic significance. N Engl J Med 1949, 241(26):1031-1036.

2. Tomlinson IP, Houlston RS: Peutz-Jeghers syndrome. J Med Genet 1997, 34(12):1007-1011.

3. Peutz JLA: Very remarkable case of familial case of polyposis of mucous membrane of intestinal tract and nasopharynx accompanied by peculiar pigmentations of skin and mucous membrane. Ned Maandschr Geneeskd 1921, 10:134-146.

4. Acea Nebril B, Taboada Filgueira L, Parajó Calvo A, Gayoso García R, Gómez Rodríguez D, Sánchez González F, Sogo Manzano C: Solitary hamartomatous duodenal polyp; a different entity: report of a case and review of the literature. Surg Today 1993, 23(12):1074-1077.

5. Kitaoka F, Shiogama T, Mizutani A, Tsurunaga Y, Fukui H, Higami $Y$, Shimokawa I, Taguchi T, Kanematsu T: A solitary Peutz-Jeghers-type hamartomatous polyp in the duodenum. A case report including results of mutation analysis. Digestion 2004, 69(2):79-82.

6. Gannon P, Dahlin D, Bartholomew L, Beahrs O: Polypoid glandular tumors of the small intestine. Surg Gynecol Obstet 1962, 114:666-672.

7. Siegel B, Yoran R: Duodenal hamartomas causing gastrointestinal bleeding. Harefuah 1978, 95:75-76.

8. Ushijima S, Nakagawa M, Kim J, Kiyohara K, Segawa Y: Duodenal hamartoma compatible with incomplete type Peutz-Jeghers syndrome: Report of a case. Stomach Intest 1986, 21:1027-1030.

9. Bott S, Hanks J, Stone D: Solitary hamartomatous polyp of the duodenum in the absence of familial polyposis. Am J Gastroenterol 1986, 81:993-994.
10. Naitoh H, Sumiyoshi $Y$, Kumashiro R, Inutsuka S, Fujita K, Yamamoto T, Murayama H: A Solitary Peutz-Jeghers type hamartomatous polyp in the duodenum. A case report. Jpn J Surg 1988, 18:475-477.

11. Rossetti G, Siringo G, Mantovani G, Musola R, Pregarz M, Volpe E, Marini E, Marino F: Duodenal hamartoma: apropos of a case report. Radiol Med 1989, 77:134-136.

12. Tanaka H, lida M, Kohrogi N, Matsui T, Yasunami Y, Yao T, Nakamura K, Fujishma M: Endoscopic removal of solitary hamartomatous polyps of the duodenum. Gastrointest Endosc 1990, 36:640-642.

13. Ichiyoshi Y, Yao T, Nagasaki S, Sugimachi K: Solitary Peutz-Jeghers type polyp of the duodenum containing a focus of adenocarcinoma. Ital J Gastroenterol 1996, 28:95-97.

14. Oncel M, Remzi FH, Church JM, Goldblum JR, Zutshi M, Fazio WW: Course and follow-up of solitary Peutz-Jeghers polyps: a case series. Int J Colorectal Dis 2003, 18:33-35.

15. Itaba S, Namoto M, Somada S, Nakamura K, Kumashiro Y, Nakamura N Yao T: Two cases of solitary Peutz-Jeghers-type hamartoma of the duodenum. Endoscopy 2006, 38(Suppl 2):E32-E33.

16. Suzuki S, Hirasaki S, Ikeda F, Yumoto E, Yamane H, Matsubara M: Three cases of Solitary Peutz-Jeghers-type hamartomatous polyp in the duodenum. World J Gastroenterol 2008, 14:944-947.

17. Jamaludin AZ, Telisinghe PU, Yapp SK, Chong VH: Solitary duodenal hamartomatous polyp with malignant transformation: report of a case. Surg Today 2009, 39:527-532.

18. Kantarcioglu M, Kilciler G, Turan I, Ercin CN, Karslioglu Y, Guvenc I, Polat Z, Bagci S: Solitary Peutz-Jeghers-type hamartomatous polyp as a cause of recurrent acute pancreatitis. Endoscopy 2009, 41(Suppl 2):E117-E118.

19. Bartholomew LG, Moore CE, Dahlin DC, Waugh JM: Intestinal polyposis associated with mucocutaneous pigmentation. Surg Gynecol Obstet 1962, 115:1-11.

20. Narita T, Eto T, Ito T: Peutz-Jeghers syndrome with adenomas and adenocarcinomas in colonic polyps. Am J Surg Pathol 1987, 11:76-81.

21. Perzin $\mathrm{KH}$, Bridge MF: Adenomatous and carcinomatous changes in hamartomatous polyps of the small intestine (Peutz-Jeghers syndrome): report of a case and review of the literature. Cancer 1982, 49:971-983.

22. Dodds WJ, Schulte WJ, Hensley GT, Hogan WJ: Peutz-Jeghers syndrome and gastrointestinal malignancy. Am J Roentgenol Radium Ther Nucl Med 1972, 115:374-377.

doi:10.1186/1752-1947-5-240

Cite this article as: Sekino et al:: Solitary Peutz-Jeghers type hamartomatous polyps in the duodenum are not always associated with a low risk of cancer: two case reports. Journal of Medical Case Reports 2011 5:240.

\section{Submit your next manuscript to BioMed Central and take full advantage of:}

- Convenient online submission

- Thorough peer review

- No space constraints or color figure charges

- Immediate publication on acceptance

- Inclusion in PubMed, CAS, Scopus and Google Scholar

- Research which is freely available for redistribution 